\title{
Sur la solution de l'équation du cinquième degré
}

par Kart Bohlin (de Stookholm).

1. Les recherches exposées dans la communication suivante sont destinées à former un complément aux mémoires précédents sur le thème dont il s'agit, publiés, en particulier, dans les «Annali di Matematica» (1) -- complément essentiel en effet par rapport aux singularités remarquées de la solution jusqu'ici considérée ('). Au surplus, par la nouvelle réduction de notre pro* blème, nous pourrons nous dispenser de la considération de la solution dite paracyclique, seulement provisoire et imparfaite au point de vue de la rigueur. Les particularitês de la théorie des équations algébriques sous la forme libre étant exposées dans les Mémoires antérieurs, il suffira d'en récapituler ici quelques points principaux, tenant aux recherches dont nous aurons à nous occuper.

Les équations normales du troisième, du quatrième et du einquième degré s'érivent, comme on sait,

$$
z^{3}+3 s z=2 \tau, \quad z^{4}+4 s z=3 \tau, \quad z^{5}+5 s z=4 \tau
$$

et pour la troisième d'elles la réduction particulière employée, dite de BRINGJERRARD, est bien connue et citée des ouvrages communs d'algèbre. La réduction à la forme libre, exposée, par exemple, dans le second des Mémoires cités des "Annali», conduit à l'équation du cinquième degré considérée dans ce qui suit:

$$
\eta^{5}+\frac{5 \eta}{u^{3}}=\frac{1}{u^{5}}-27
$$

(1) I. Suv l'équation algébrique du cinquième degré, "Annali di Matematica pura ed applicata", Serie IV, Tomo XI, 1982-33; II. Sur la solution de l'équation générale du cinquième degré, "Annali di Matematica", Serie IV, Tomo XII, 1983-34.

(2) Zusätze und Erlüuterungen zur Lösung der algebraischen Gleichung fünften Grades auf Grundlage des Racinals, "Arkiv für Matematik, Astronomi o. Fysik. ", K. Vetenskapsa* kademien, Stockholm, Band $25 \mathrm{~A}$, N. ${ }^{\circ} 6,193 \overline{.}$. 
Une méthode directe de former la racine prineipale d'une équation de forme libre e'est de développèr la racine dans les environs de $\frac{1}{u^{5}}=\infty$ selon les puissances de la quantité $u$. Nous parviendrons par des approximations successives à nne série qui, pour l'équation du troisième degré, se troure interrompue dès les deux premiers termes

$$
\eta=\frac{1}{u}-1
$$

et qui, pour celle du quatrième degré, est déjà infinie, mais réductible anx expressions algébriques finies

$$
\eta=-1+\sqrt{\frac{1}{u^{2}}-1} ; \quad \eta=+1+i \sqrt{\frac{1}{u^{2}}+1}
$$

Les résultats, obtenus selon cette méthode pour l'équation (1), sont donnés dans les Mémoires antérieurs. En premier lieu nous tronverons

$$
\eta=\frac{1}{u} A-B-u C-u^{2} D *
$$

ThÉonìne I. - Tous les termes contenant le facteur $u^{3}$, qui suiveraient dans cette expression, s'evanouissent.

Les termes ultérieurs se réunissent aux quatre précédents, en sorte que les coëfficients $A, B, C, D$ soient des séries procédant suivant $u^{5}$. Pour chaque équation algébrique on aura en général à employer une résolvante de degré inférieur à l'équation originale. Pour l'équation (1) la résolvante valant pour les valeurs de $u^{5}$ est bien de la forme

$$
\alpha^{3}+\alpha^{2}+\alpha+3 u^{5}=0
$$

et possède les mèmes points de raeines égales que l'équation originale (1), savoir les points

$$
\frac{1}{u^{5}}=7+4 i \sqrt{2}
$$

On peut alors développer les coefficients $A, B, C, D$ suivant les puissances de $\alpha$; il sera même plus avantagenx encore de choisir la quantité $\beta$, definie par

$$
\beta=\alpha+\alpha^{2},
$$

pour élément du développement. C'est en réduisant ces séries en forme de produits infinis suivant les facteurs $(1+\beta),\left(1+\beta^{2}\right), \ldots$ que la rélation fondamentale - 
Théorène II. $A D=B C$

- se tronve établie. Introduisons les notations:

$$
\delta=\frac{\alpha}{3} ; \quad \gamma=\frac{\beta}{3},
$$

et posons

$$
s=A D=B C .
$$

Ainsi qu'il a été démontré dans le Mémoire II, on a alors

$$
s=\gamma-2 \gamma^{2}+4 \gamma^{3}-3 \gamma^{4}
$$

En posant encore

$$
p=\frac{A C}{D}
$$

l'expression de la quantité $p$ est bien

$$
p=1+3 \delta+5 \delta^{2}-4 \delta^{3}-4 \delta^{4},
$$

et notre développement, dit racinal, sera ainsi:

$$
\eta=\frac{1}{u} A-\frac{A^{2}}{p}-u \frac{p}{A^{2}}(1-s)-u^{2} \frac{1}{A}(1-s) .
$$

Poux ce qui concerne la quantité $A$ dans cette formule, tandis qu' il ne parait pas possible d'en tronver un développement en série convenable, on obtient au contraire une expression symétrique de la einquième puissance d'elle, à savoir

$$
A^{5}=1+6 \delta+22 \delta^{2}+36 \delta^{3}+22 \delta^{4}+6 \delta^{5}+\delta^{6} .
$$

Nous désignons la racine cinquiòme de ce polynome par $A_{\delta}$, pour faire ressortir la connexion fonctionelle dè $A$ avec la quantité $\delta$.

Il existe encore une autre représentation de $A$, fondée sur l'expression Ogalement symétrique suivante:

$$
A^{5}=1+6 s+16 s^{2}+52 s^{3}+16 s^{4}+6 s^{5}+s^{6} .
$$

Désignons la racine cinquième de ce polynome en $s$ par $A_{s}$, pour faire ressortir la connexion fonctionelle entre $A$ et la quantité $s$, définie ci-hant.

Cette quantité $A_{s}$ a reçu moins d'attention, d'une part parceque la quantité $A_{\delta}$ paraît suffisante pour le but, et d'autre part parceque l'analogue de la quantité $s$, dans le cas de l'équation du quatrième degré, consiste en une constante, figurant en facteur $\frac{1}{2}$ du dernier terme de l'expression trinôme de la racine dans ce cas. Néanmoins l'introduction de la quantité $A_{s}$ à côté 
de $A_{\delta}$ paraît bien propre à contribuer à certaines simplifications, que nous allons succinctement considérer, si même leure signification ne soit pas d'importance plus profonde.

2. Racines sur l'axe réel des arguments $\frac{1}{u^{5}}$. - La formule (2) de la racine amène pour des valeurs assez grandes de $\frac{1}{u^{5}}-$ soit depuis $\frac{1}{u^{5}}=27$ $\grave{a} \frac{1}{u^{5}}=\infty$ - l'accord presque parfaît en sept décimales. Par exemple, s'obtient, pour

$$
\frac{1}{u^{5}}=54, \quad \eta=+0.4925960, \quad \text { valeur vraie }:+0.4925958 .
$$

A mesure que le paramètre s'approche de l'origine $\frac{1}{u^{5}}=0$, l'accord se trouve réduit. La formule employée conduit pourtant au point $\frac{1}{u^{5}}=3$ à la valeur de la ràcine

$$
\eta=-1.581439, \quad \text { valeur vraie }:-1.55018461=-3^{3 / 5} .
$$

Au lieu du proposé, fait dans le mémoire précédent, de faire usage d'une paire d'équations du quatrième degré, poux arriver à l'accord parfait dans ce point, nous allons proposer le procédé qui suit. Mais nous faisons remarquer d'avance que, selon cette méthode ainsi que par l'autre, il restera des écarts pour des arguments intermédiaires tels que $\frac{1}{u^{5}}=7$ et $\frac{1}{u^{5}}=8$; et, si mème par quelque méthode il réussit d'anéantir la différence, ayant lieu poux $\frac{1}{u^{5}}=7$, la différence au point voisin $\frac{1}{u^{5}}=8$ n'est pas remuée. Voici cependant en quoi consiste le procédé nouveau. Soient

$$
\eta_{i}, \quad \eta_{s}
$$

deux valeurs de la racine obtenus en employant la formule (2) et respectivement $A=A_{\delta}$ et $A=A_{s}$.

Posons

$$
\eta_{0}=\frac{\eta_{\delta}+p_{s} \eta_{s}}{1+p_{s}}
$$

et essayons de déterminer la quantité $p_{s}$ de manière que l'accord

$$
\eta=\eta_{0}
$$

aura lieu pour $\frac{1}{u^{5}}=3$ et pour $\frac{1}{u^{5}}=27$. 
Nous obtenons au moyen de notre formule (2), pour $\frac{1}{u^{5}}=3$

$$
\eta_{\delta}=1.5814388, \quad \eta_{s}=1.4522155, \quad \text { valeur vraie }: \eta=1.5518462,
$$

d' où se déduit tout de suite

$$
p_{s}=+0.29702
$$

En écrivant

$$
p_{s}=p_{0}\left[1-100^{+}-\frac{1}{(100)^{2}}-\frac{1}{(100)^{3}}+\ldots\right],
$$

avec

$$
p_{0}=\frac{1}{3} \cdot \frac{1}{1+\left(u^{5}\right)^{2}}=\frac{3}{10}
$$

nous aurons

$$
p_{s}=0.29702970297 \ldots,
$$

ce qui d'après (o) nous donne $\eta=1.551846$, tout en accord avec la valeur vraie de $\eta$ ei-haut.

$$
\begin{aligned}
& \text { Pour } \frac{1}{u^{5}}=27 \text { s'obtient } \\
& \qquad \begin{aligned}
& \log p_{s}=9.5222824 \log \eta_{s}=2.09671 \\
& \log p_{s}=9.52228 \\
& p_{s} \eta_{s}=+41.7 \\
& \eta_{s}=-42.0 \text { septième décimale } \\
& \\
& \eta_{0}=-0.000000 .3 .
\end{aligned}
\end{aligned}
$$

La formule générale de $p_{s}$ employée est

$$
p_{s}=\frac{1}{3} \cdot \frac{1}{1+\left(u^{5}\right)^{2}} \cdot \frac{1+2\left(u^{5}\right)^{2}}{\left[1+\left(u^{5}\right)^{2}\right]^{2}} \cdot \frac{1}{1-\left[\frac{\left(u^{5}\right)^{2}}{1+\left(u^{5}\right)^{2}}\right]^{4}} .
$$

Nous avons eu soin de calculer d'après cette méthode les vauleurs de $\eta_{0}$ suivantes, intermédiaires à $\frac{1}{u^{5}}=3$ et $\frac{1}{u^{5}}=27 ; \frac{1}{u^{5}}=+5,+7,+8,+10.125$, +14 et pour $\frac{1}{u^{5}}=-9$ - points, on les valeurs vraies des racines sont données dans le Mémoire I. Sans entrer en particularités dans les calculs, il suffira de constater ici que l'accord s'est trouvée plus grand dans tous les cas, en employant le procédé nouveau relatif à la formule (5), qu' en employant 
respectivemont $\eta_{\delta}$ on $\eta_{s}$ seules an calcul d'après la formule (2). Voici les valeurs calculées de $\eta_{0}$ et, à còté d'elles, le valeurs vraics $\eta$ des racines.

\section{TABLEAU $\mathrm{I}$.}

\begin{tabular}{crr}
1 & \multicolumn{1}{c}{$\eta_{0}$} & $\eta$ \\
$u^{5}=3$ & -1.5518462 & -1.5518462 \\
5 & -1.3383009 & -1.3427982 \\
7 & -1.1277686 & -1.1299153 \\
8 & -1.0247066 & -1.0259724 \\
10.125 & -0.8223538 & -0.8226765 \\
14 & -0.5319309 & -0.5319505 \\
27 & 0.0000000 & 0.0000000 \\
-9 & -2.4082272 & -2.4082245.
\end{tabular}

Les écarts se trouvent notés dans la troisième colonne du Tableau II; (7-a).

Ainsi qu'il va ressortir du n. ${ }^{\circ} \bar{y}$ ci-après, il y'aura lieu de considérer encore les éléments suivants

$$
\eta_{\delta s} \text { et } \eta_{s \delta},
$$

formés en prenant dans l'expression (2) denx termes en $A_{\delta}$ et les deux autres de $A_{s}$ ou inversement. Nous pouvons alors faire l'bypothèse

$$
\eta-\eta_{0}=\frac{\eta_{\delta s}-\eta_{0}+f\left(\eta_{s \delta}-\eta_{0}\right)}{1+f}
$$

ou, ce qui revient au mème,

$$
\eta=\frac{\eta \delta s+f \eta_{s \delta}}{1+f}
$$

En déterminant la quantité $f$ d'après la formule linéaire

$$
f=a+\frac{b}{u^{\tilde{3}}}
$$

où il est facile de déduire, d'après les données pour $\frac{1}{u^{5}}=3$ et $\frac{1}{u^{5}}=27$, les coefficients

$$
a=1+\frac{4 \cdot 3^{3}}{10^{3}}=1.1081 ; \quad b=3^{3} \cdot \frac{4^{2}+3^{3}}{10^{4}}=0.0387
$$

on aura en effet des écarts encore amoindris, se trouvant dans la quatrième colonne du Tableau suivant. 


\section{TABLEAU II.}

$\begin{array}{crrr}\frac{}{4} \eta-\eta_{0} \text { formule }(8) & \eta-\eta_{0} \text { formule }(5) & \text { écarts }(8)-(5) \\ u^{5} & = & 0.000000 & 0.000000 \\ 3 & 0.000000 & -0.004497 & +0.000115 \\ 7 & -0.004606 & -0.002147 & -0.000869 \\ 8 & -0.001278 & -0.001266 & -0.000683 \\ 10.125 & -0.000583 & -0.000323 & -0.000207 \\ 14 & -0.000011 & -0.000020 & -0.000009 \\ 27 & 0.000000 & 0.000000 & 0.000000 \\ -9 & +0.000216 & +0.000002 & -0.000214 .\end{array}$

Ainsi les écarts se trouvent d'après cela très réduits, outre pour $\frac{1}{u^{5}}=-9$. Il paraît que la formule (5) soit en quelque sorte à préférer.

Pour atteindre un accord parfait, il faudra pourtant, comme doit ressortir de ces recherches, faire usage de quelque autre procédé.

3. Approximations sur l'axe réel des arguments $\frac{1}{u^{5}}$. - Afin d'arriver au but, il faudra encore avoir. recours à notre équation originelle

$$
\eta^{5}+\frac{5 \eta}{u^{3}}=\frac{1}{u^{5}}-27
$$

Soit $\left(\eta_{0}\right)$ une valenr approchée de la racine en quelque point, par exemple obtenue par la méthode décrite das le n. ${ }^{0}$ précédent, et posons

$$
\eta=z \cdot\left(\eta_{0}\right) \text {. }
$$

Alors nous pouvons nous assurer que la valeur de $z$ soit à peu près égale à l'unité. Nous aurons l'équation de la forme normale

$$
z^{5}+5 s z=4 \tau
$$

avec

$$
5 s=\frac{5}{\left(\eta_{0}\right)^{4}} \cdot \frac{1}{u^{3}} ; \quad 4 \tau=\frac{\frac{1}{u^{5}}-27}{\left(\eta_{0}\right)^{5}},
$$

et il s'agit de réduire cette équation à la forme libre.

Ainsi qu'il fut exposé dans les Mémoires antérieurs, nous avons à ce but la résolvante

$$
\left.w^{4}+4 \tau w=3 s^{5}\right)_{s}
$$


et les substitutions

$$
u_{1}=\frac{\left(\frac{w}{3}\right)^{4 / 5}}{s^{1 / s}} ; \quad \zeta_{1}=\frac{\ddot{z} \cdot\left(\frac{w}{3}\right)^{1 / 5}}{s^{1 / s}}
$$

qui amènent l'équation nouvelle de forme libre

$$
\zeta_{1}^{5}+5 u_{1} \zeta_{1}=1-27 u_{1}^{5}
$$

ou bien, en posant

$$
\zeta_{1}=\eta_{i} u_{i}
$$

l'équation

$$
\eta_{1}^{5}+\frac{5 \eta_{1}}{u_{1}^{3}}=\frac{1}{u_{1}^{5}}-27
$$

c.-à-d. l'équation primaire, pourtant à d'autres valours de $\frac{1}{u^{3}}$ et $\frac{1}{u^{5}}$. On peut déterminer, d'après la formule (2) du n. 1 la racine nouvelle $\eta_{1}$. Cela étant, nous aurons

$$
z=\eta_{1} u_{1} \cdot \frac{s^{1 / s}}{\left(\frac{w}{3}\right)^{\eta_{15}}}
$$

après quoi s'obtient la valeur de la racine de l'équation originelle, en seconde approximation, - soit $\eta$ - selon (9), c.-àd-d.

$$
\eta=\approx \cdot\left(\eta_{0}\right) \text {. }
$$

Quelques exemples vont prouver l'effet de ce procédé.

Exemple 1. $\frac{1}{u^{5}}=7$. - Selon le Tablean $(7-a)$ ei-haut nous avons la différence $\eta-\eta_{0}=-0.001278$, de quoi s'ensuit, en considérant la valeur primaire $\eta_{0}=-1.1277686 \mathrm{du}$ Tableau (7), la valeur

$$
\left(\eta_{0}\right)=-1.129047
$$

pour la valeur approchée de la racine, devant ètre employée. Il s'ensuit

$$
\log s=0.2362116 ; \quad \log \tau=0.4354110 ; \quad \log w=9.912469 .
$$

Il doit ètre utile de donner un peu de particularités du caleal dans cet exemple. Ainsi qu'il doit ètre connu, l'équation du quatrième degré (11) admet elle-mème la résolvante du troisième degré

$$
w_{1}^{3}+3 \tau_{1} w_{1}=2 s_{1}^{2},
$$

dont la solution est donnée d'apres les formules connues pour les racines 
d'ane équation du troisième degré. O'est ainsi que nous obtenons

$$
\frac{1}{u_{i}^{5}}=567.0005 \equiv 7.81
$$

et

$$
\log z=0.0003337 \text {. }
$$

Nons avons obtenu déjà

$$
\log \left(\eta_{n}\right)=0.0527118_{n},
$$

de sorte qu'on obtient encore

$$
\log \eta=0.0530455_{n}
$$

c.-à-d.

$$
\eta=z \cdot\left(\eta_{0}\right)=-1.129914, \quad \text { valeur vraie }:-1.129915 \text {. }
$$

Exencle 2. $\frac{1}{u^{5}}=8$. - Dans ce cas la solution primaire (Tableau I) a donné $\eta_{0}=-1.0247066$, d'où, en considérant les écarts du Tableau (7-a)

$$
\left(\eta_{0}\right)=-1.0252898
$$

quantité devant être employée comme point de départ. Tout comme dans l'exemple précedent, on trouvera successivement

$$
\log s=0.4984672, \quad \log \tau=0.6284601, \quad \log w=0.6485849
$$

ot puis

$$
\frac{1}{u_{1}^{5}}=350.6905, \quad \log z=0.0002894
$$

Nous avions

et l'on déduit par suite

$$
\log \left(\eta_{0}\right)=0.0108467_{n}
$$

$$
\eta=\left(\eta_{0}\right) \cdot z=-1.025973, \quad \text { valeur vraie: }-1.025972 \text {. }
$$

Nous voyons, par ces exemples, comment par le procédé employś les arguments primaires $\left(\frac{1}{u^{5}}=7 ; \frac{1}{u^{5}}=8\right)$ viennent ètre transposés à des point plus lointains $\frac{1}{u_{1}^{5}}=567$ ou $\frac{1}{u_{1}^{5}}=350.6905$, et que désormais ils appartiennent au domaine racinal ou extérieur, s'étendant à l'infini, tandis qu' on pent dire que les points primaires $\frac{1}{u^{5}}=7$ et $\frac{1}{u^{5}}=8$ mèmes appartiennent à un domaine interne, se trouvant aux environs plus ou moins étendus autour de l'origine, domaine où, comme nous l'avons vu, l'application de la formule racinale (2) est en défaut. Les exemples font voir encore que le procédé employé conduit 
bien aux valeurs vraies des racines cherchées. On pourrait être d'avis que le procédé pourrait être poursuivi par des approximations successives. Mais nous allons voir qu'il n'est pas ainsi, et qu'il n' existe pas d'autres approximations successives.

Ajoutons encore l'exemple suivant.

Exemple 3. $\frac{1}{u^{5}}=54$. - Dans ce troisième cas la solution primaire était

$$
\eta=+0.4925960
$$

tout en accord avec la valeur vraie, +0.4925958 . Si pourtant nous nous mettons à l'application du procédé de la solution secondaire, dans ce cas

$$
\left(\eta_{0}\right)=+0.4925960
$$

nous sommes conduits, tout comme dans les exemples précédents, aux ré. sultats suivants

$$
\log s=0.2694727, \quad \log \tau=2.3668493, \quad \log w=0.9896368
$$

et puis

$$
\begin{aligned}
\frac{1}{u_{1}^{5}}=53.99993 \quad & \log z=0.0000002 \\
& \log \left(\eta_{0}\right)=9.6524909 \\
& \log \eta=9.6924911 \\
\eta= & +0.492596 .
\end{aligned}
$$

Ainsi la seconde solution amène l'argument de départ $\left(\frac{1}{u^{5}}=54\right)$ et la valeur primaire mème de la racine $\eta$.

4. Sur les singularités de la solution. Les points isolines. - Tandis que il faut supposer que les points sur l'axe réel ou dans ses environs, auxquels tout d'abord nous avons adjugé une solution «paracyclique », viendront se transposer à l'aide de la méthode nommée au domaine racinal, il y aura de l'interêt de considérer à part les points isolines proprement dits. Ce sont en particulier les points, où la solution (2) prend des valeurs infinies. C'est ce qui a lieu pour $A_{\delta}=0$ et pour $p=0$. Considerons les $\delta$-isolines. Ce sont les racines de l'équation (')

$$
A_{\delta}=0
$$

(1) Zusätze und Evläuterungen zur Lösung der algebraischen Gleichung fünften Grades auf Grundlage des Racinals; "Arkiv fux Matematik, Astronomi o. Fysik", K. Vetenskapsa* kademien, Stockholm, Band $25 \mathrm{~A}, \mathrm{~N} .{ }^{\circ} 6,1935$. 
savoir

$$
\begin{array}{ll}
\delta_{1}=-1.6911970, & \delta_{3}, \delta_{4}=-0.1498669 \pm i 0.2554175 \\
\delta_{2}=-0.5912970, & \delta_{5}, \delta_{6}=-1.7088867 \mp i 2.9124605,
\end{array}
$$

auxquelles corréspondent

$$
\begin{array}{cl}
\text { pour } \delta_{1}, \delta_{2} & \frac{1}{u^{5}}=+0.027 \text { et }+0.713, \\
\gg \delta_{5}, \delta_{6} & \text { des valeurs encore plus petites, } \\
》 \delta_{3}, \delta_{4} & \frac{1}{u^{5}}=+15.23295 \pm i 10.49492
\end{array}
$$

Les quatre premiers de ces points appartiennent au domaine des solutions "paracycliques». Mais quant aux deux derniers il y aura de difficulté d'appliquer une solution de cette sorte.

Si, comme nous avons essayé, on introduit encore l'expression

$$
A_{s}=1+6 s+16 s^{2}+52 s^{3}+16 s^{4}+6 s^{5}+s^{6}
$$

il faut aussi considérer les s-isolines, tenant à l'équation

$$
A_{s}=0
$$

du sixième degré, qui toutefois, comme au cas antérieur, se rèduit au troisième degré. En effet, en posant

$$
s+\frac{1}{s}=x \quad \text { et } \quad x=y-2,
$$

elle se réduit ì

$$
y^{3}+y+30=0
$$

avec la racine réelle $y=-3$. Il n'y a pas lieu d'exposer ici les valeurs de $\frac{1}{u^{5}}$ corréspondantes $q u$ 'on en déduit. De même pour les $p$-isolines on aura à résoudre l'équation

$$
p=1+3 \delta+5 \delta^{2}-4 \delta^{3}-4 \delta^{4}=0
$$

ce qui conduit, par la substitution $\delta=y-\frac{1}{4}$, à l'équation

$$
y^{4}-\frac{26}{16} y^{2}-\frac{39}{256}=0
$$

ō. Sur une représentation de Ia racine dans les points isolines. - II s'agit des points

$$
\frac{1}{u^{5}}=15.2329+i 10.4949
$$

du n. ${ }^{\circ}$ précédent, où l'expressiou principale (2) devient infinie. 
Pour l'éviter on pourrait introduire dans cette formule la quantité $A_{s}$ au lieu de $A_{\S}$, ce qui donne

$$
\begin{aligned}
& \frac{1}{u} A_{s}=-2.4366-i 0.0584 \\
& -\frac{A_{s}{ }^{2}}{p}=-1.7306+i 3.8982 \\
& -u \frac{p}{A_{s}{ }^{2}}(1-s)=-0.1123-i 0.1664 \\
& -u^{2} \frac{1}{A_{s}}(1-s)=+0.3443-i 0.0698 .
\end{aligned}
$$

Puisque la valeur vraie de la racine, dans le point cunsidéré, est

$$
\eta=-0.25161+i 0.48639
$$

la représentation n'étant pas atteinte par l'expression complète en $A_{s}$, on peut introduire la représentation $\eta_{\delta s}$, qui, à cause de $A_{\delta}=0$, se réduit aux deux derniers termes

$$
\eta_{\delta s}=-u \frac{p}{A_{s}{ }^{2}}(1-s)-u^{2} \frac{1}{A_{\delta}}(1-s)
$$

ce qui donne

$$
\eta_{\delta s}=+0.2330-i 0.2362 \text {. }
$$

Soit $\eta_{0}=\eta_{\delta s} . L$ 'expression diffère pourtant encore de

$$
\eta=-0.2516+i 0.4864
$$

mais il est facile de trouver l'expression

$$
\eta=\frac{\eta_{0}}{2}+\sqrt{\frac{\eta_{0}^{2}}{4}+i k_{0}}
$$

qui, pour $k_{0}=\frac{3}{10}$, fait représenter la racine. On pourra mème donner à la quantité $k_{0}$ une forme, qui pour l'axe réel se réduit à zéro et aux points isolines retient la valeur de $3 / 10$ à peu près. Si l'on pose

$$
\frac{1}{u^{5}}=\alpha+i \beta=\lambda e^{i \omega}
$$

cela est atteint par la formule

$$
k_{0}=\sin ^{2} \varphi .
$$

Pour les isolines serait à peu près

$$
\frac{\beta}{\alpha}=\frac{2}{3}
$$

et $k_{\vartheta}=4 / 13$. Au surplus nous aurions, pour $\varphi=90^{\circ}, k_{0}=1$. 
Pour les points des racines égales

$$
\frac{1}{u^{5}}=7+i \sqrt{32}
$$

on aurait de cette manière $k_{0}=0.39505$ et la formule (12) donnerait

$$
\eta=1.96338-i 0.1382
$$

tandis que, en réalité,

$$
\eta=1.7796-i 1.4492 .
$$

Évidemment ainsi la formule (12) doit être toute particulière pour les points isolines.

6. Les points de racines égales. - Nous avons entrepris d'éprouver encore la formule (5)

$$
\eta_{0}=\frac{\eta_{\delta}+p_{s^{\prime} l s}}{1+p_{s}}
$$

pour les points

$$
\frac{1}{u^{5}}=7+4 i \sqrt{2}
$$

des racines égales.

Quoique, ainsi qu'il y aurait lieu de prévoir, l'accord parfait avec la réalité n'est pas atteint par cela, le résultat confirme pourtant l'utilité de la formule (5) à un certain degré, aussi pour des valeurs complexes, comme celles rélatives aux racines égales.

La résolvante a dans ce cas la forme

$$
\alpha^{3}+\alpha^{2}+\alpha=-3 u^{5}=-\frac{3}{7+4 i \sqrt{2}}
$$

ef fournit la valeur

$$
\alpha=\frac{-1+2 i \sqrt{2}}{2}
$$

devant être employée. On déduit de la manière usuelle successivement les quantités $\beta, \gamma, \delta$ ainsi que $p$ et $\delta$. Ayant obtenu à la suite $A_{\delta}$ et $A_{s}$, on a formé les valeurs

$$
\eta_{\delta}=+1.98826+i 1.47790, \quad \eta_{s}=+1.26052+i 2.18996 .
$$

De plus, au moyen de la formule (6), s'obtient

$$
p_{s}=[9.51813]+i[7.60732] \text {. }
$$

Évidemment on aurait pu se contenter avec la valeur $p_{s}=1 / 3$. 
Notre formule (5) donne ainsi

$$
\eta_{0}=\frac{\eta_{\delta}+p_{s} \eta_{s}}{1+p_{s}}=1.8061-i 1.6528
$$

à quoi on pent comparer la valeur vraie

$$
\eta=1.7796-i 1.4492 \text {. }
$$

Le calcul donne ainsi la partie réelle de l'approximation suffisante, mais il vaudra mieux d'apporter la partie imaginaire de l'expression simple de

$$
\eta_{\delta}=1.98826-i 1.47790
$$

en mettant pour point de départ du calcul suivant

$$
\left(\eta_{n}\right)=1.8061-i 1.4779 \text {. }
$$

7. Les points de racines égales (suite). - Il va se trouver que le point de départ de la seconde approximation soit arbitraire. Néanmoins nous avons employé la valeur indiquée à la fin $d u n .{ }^{\circ}$ précédent. Le résultat du calcul soit donné tout d'abord. On a trouvé

$$
\eta=1.7794-i 1.4493
$$

la valeur vraie étant

$$
\eta=1.7796-i 1.4492 \text {. }
$$

Ainsi cot exemple vérifie de nouveau le procédé employé. Il pent ètre utile d'en adjoindre ici un exposé succinct. Le calcul fut conduit de la même manière que dans le n. ${ }^{\circ} 3$, savoir en mettant dans l'équation originelle

$$
\eta^{5}+\frac{5 \eta}{u^{3}}=\frac{1}{u^{2}}-27
$$

la substitution

par où l'équation

$$
\eta=\left(\eta_{0}\right) \cdot z
$$

$$
z^{5}+\check{5} s z=4 \tau
$$

est obtenue, avec

$$
5 s=\frac{\tilde{o}}{\left(\eta_{0}\right)^{4} u^{3}} ; \quad 4 \tau=\frac{\frac{1}{u^{5}}-27}{\left(\eta_{0}\right)^{5}} .
$$

Au cours du calcul il s'est trouvé que

$$
\tau^{4}+s^{5}=0
$$

relation simplifiant la question, qui désormais peut ètre traitée, sans des 
calculs numériques, comme il suit. À traduire à la forme libre sert la résolvante

$$
w^{4}+4 \tau w=3 s^{5} \%
$$

et les substitutions

$$
u_{0}=\frac{\left(\frac{w}{3}\right)^{4 / s}}{s^{1 / s}} ; \quad \zeta_{0}=z \cdot \frac{\left(\frac{w}{3}\right)^{1 / s}}{s^{1 / s}},
$$

par où s'obtient l'équation

$$
\zeta_{0}^{5}+5 u_{0} \zeta_{0}=1-27 u_{0}^{5}
$$

Pour la solation de la résolvante du quatrième degré il sera avantageux de mettre

$$
w=z_{1} ; \quad \tau=s_{1} ; \quad s^{5 / s}=\tau_{1} .
$$

L'équation prend avec cela la forme

$$
z_{1}^{1}+4 s_{1} z_{1}=3 \tau_{1} \text {. }
$$

Elle possède de nouvean la rósolvante

$$
w_{1}^{3}+3 \tau_{1} w_{1}=2 s_{1}^{2},
$$

et se trouve, par les substitutions

$$
u_{1}=\frac{\left(\frac{w_{1}}{2}\right)^{3 / 2}}{s_{1}^{1 / 9}} ; \quad \zeta_{1}=z_{1} \cdot \frac{\left(\frac{w_{1}}{2}\right)^{1 / 4}}{s_{1}{ }^{1 / 2}},
$$

réduite ì la forme libre

Soit

$$
\zeta_{1}^{4}+4 u_{1} \zeta_{1}=1-4 u_{1}^{4}
$$

$$
\zeta_{1}=u_{1} \eta_{1}
$$

pour transformer aux deux équations séparées:

$$
\eta_{1}^{2}+2 \eta_{1}=\frac{1}{u_{1}^{2}}-2, \quad \eta_{1}^{2}-2 \eta_{1}=-\frac{1}{u_{1}^{2}}-2,
$$

de manière que, par exemple,

$$
\zeta_{1}=\eta_{1} u_{1}=-u_{1}+\sqrt{1-u_{1}^{2}}
$$

soit l'une des solutions.

Pour la résolvante du troisième degré nous avons la solution bien connue

$$
w_{1}=\left[s_{1}^{z}+\sqrt{s_{1}^{4}+\tau_{1}^{3}}\right]^{2 / 3}+\left[s_{1}^{z}-\sqrt{s_{1}^{4}+\tau_{1}^{3}}\right]^{1 / s},
$$

ou bien, d'après les désignations introduites,

$$
w_{1}=\left[\tau^{2}+\sqrt{\tau^{4}+s^{5}}\right]^{1 / 3}+\left[\tau^{2}-\sqrt{\tau^{4}+s^{5}}\right]^{1 / 3} .
$$


On trouve ainsi, par suite de la condition

$$
\tau^{4}+s^{5}=0
$$

les valeurs

$$
w_{1}=2\left(\tau^{2}\right)^{1 / 3} ; \quad u_{1}=\frac{\tau^{1 / 2}}{\tau^{1 / 2}}=1 ; \quad \zeta_{1}=-1
$$

puis encore

et enfin

$$
z_{1}=-\tau^{1 / s} ; \quad w=z_{1}=-\tau^{1 / s}
$$

$$
\frac{1}{u_{0}^{5}}=-81 ; \quad z=-\eta_{0} \cdot \frac{\tau^{1 / 5}}{3^{3 / 5}} \text {. }
$$

A la détermination de $\eta_{i}$ servirait l'équation

$$
\eta_{0}^{5}+\frac{5 \eta_{0}}{u_{0}^{3}}=\frac{1}{u_{0}^{5}}-27
$$

mais, la valeur de cette racine est connue (1) et égale à

de sorte qu'on obtient

$$
\eta_{0}=+3^{m / 3}
$$

$$
z=-\tau^{1 / 5}
$$

et, en considérant la rélation

$$
4 \tau=\frac{\frac{1}{u^{5}}-27}{\left(\eta_{0}\right)^{5}},
$$

on trouve

$$
\tau^{1 / 5}=-\frac{(-\tilde{\rho}+i \sqrt{2})^{1 / 5}}{\left(\eta_{0}\right)}
$$

On a ainsi

$$
\left.\eta=(5-i \sqrt{2})^{1}\right\}_{\bar{s}}
$$

ce qui, en effet, est la valeur de chacune des racines égales. La valeur de la racine principale s'en déduit en multipliant par

$$
\lambda=-1.65063
$$

racine de l'équation

$$
\lambda^{3}+2 \lambda^{2}+3 \lambda+4=0
$$

ainsi qu'il est facile de se convaincre. Notre résultat devient par cela

$$
\eta=1.7794-i 1.4493
$$

ainsi qu'il fut noté à l'introduction du $n .^{\circ}$ présent.

(1) "Annali di Matematica", Tomo XI, pag. 270. 
8. L'invariante $x_{\text {. }}$ - Nous pourrons tout d'abord introduire une quańtité $x$, définie par

$$
x=\frac{s^{5}}{\tau^{4}}=\frac{1}{\left(u^{5}\right)^{3}} \cdot \frac{4^{4}}{\left(\frac{1}{u^{5}}-27\right)^{4}}
$$

é indépendante du choix de la valeur de $\left(\eta_{0}\right)$. Cette quantité admet des simplifications en cas des racines égales:

$$
\frac{1}{u^{5}}=7+4 i \sqrt{2}
$$

et précisément dans ces points on a

$$
x=-1 \text {. }
$$

La quantité $x$ se trouve, en général, propre aux développements dont il s'agit.

On peut se demander quels points $\frac{1}{u^{5}}$ viennent correspondre à une valeur $x$ d'avance donnée. Soit

$$
\eta^{5}+\frac{5 \eta}{u^{3}}=\frac{1}{u^{5}}-27
$$

l'équation donnée et

$$
z^{5}+5 s z=4 \tau
$$

la transformée, provenant en mettant $\eta=\left(\eta_{0}\right)$ s. Puisque la quantité $\left(\eta_{0}\right)$ s'en va de l'expression de $x$, nous sommes autorisés de prendre $\left(\dot{\eta}_{0}\right)=1$. En mettant $\frac{1}{u^{5}}=\varepsilon$ la quantité $x$ s'écrit

$$
x=\frac{256 \varepsilon^{3}}{(\varepsilon-27)^{4}} \text {. }
$$

On obtient ainsi une équation du quatrième degré en $\varepsilon$, à savoir

$$
\varepsilon^{4}-\left(108+\frac{256}{x}\right) \varepsilon^{3}+6 \varepsilon^{2}(27)^{2}-4 \varepsilon(27)^{3}+(27)^{4}=0 .
$$

Par l'application de la substitution

$$
\frac{27}{\varepsilon}=x+1=27 u^{5}
$$

l'équation se réduit à la forme normale

$$
x^{4}+4 s_{0} x=3 \tau_{0},
$$

avec les valeurs suivantes des coefficients

$$
s_{0}=-\left(\begin{array}{l}
4 \\
3
\end{array}\right)^{3} \frac{1}{x} ; \quad \tau_{0}=\left(\begin{array}{l}
4 \\
3
\end{array}\right)^{3} \frac{1}{x} .
$$


En effectuant la solution nous aurons à considérer la résolvante

$$
w_{0}^{3}+3 \tau_{0} w_{0}=2 s_{0}^{2}
$$

et à mettre, d'après le procédé de solution de l'équation du quatrième degré,

$$
u_{0}=\frac{\left(\frac{w_{0}}{2}\right)^{3 / 4}}{s_{0}{ }^{1 / 2}} ; \quad w_{0}=\left(\frac{4}{3}\right)^{2} \frac{1}{\kappa^{2 / 3}} R
$$

où nous aurons bien

$$
R=[1+\sqrt{1+x}]^{1 / 3}+[1-\sqrt{1+x}]^{1 / s} .
$$

Sans entrer dans les particularités des déductions, les résultats suivants sont à noter. On aura

$$
u_{0}=-i\left(\frac{R}{2}\right)^{9 / 4} ; \quad x=i \frac{4}{3} \frac{1}{x^{1 / 3}}\left(\frac{1}{\left(\frac{R}{2}\right)^{3 / 4}} \cdot \zeta_{0},\right.
$$

où $\zeta_{0}$ soit l'une des racines des equations séparées

c.-à-d.

$$
\zeta_{0}^{2}+2 u_{0} \zeta_{0}=1-2 u_{0}^{2} ; \quad \zeta_{0}^{2}-2 u_{0} \zeta_{0}=-1-2 u_{0}^{2}
$$

$$
\zeta_{0}=-u_{0}+i \sqrt{1-u_{0}^{2}} ; \quad \zeta_{0}=+u_{0}+i \sqrt{1+u_{0}^{2}}
$$

Dans notre cas ce sont les racines

$$
\zeta_{0}=u_{0} \pm i \sqrt{1+u_{0}^{2}}
$$

qu'il faut employer. L'une de ces racines réconduit à la valeur primaire de $\frac{1}{u^{5}}$, l'autre cependant à un point nouvean $\frac{1}{u^{5}}$. Au contraire, les racines

$$
\zeta_{0}=-u_{0} \pm \sqrt{1-u_{0}^{2}}
$$

vont conduire à des résultats étrangers à la questions. Nous n'insisterons pas de plus sur ces circonstances, parceque l'équation (15) ne fut employée que pour le controle.

9. Sur la solution de l'équation proposée: $\eta^{5}+\frac{5 \eta}{x^{3}}=\frac{1}{u^{5}}-2 \%$ - L'équation importante (15) du n. ${ }^{\circ}$ précédent, tout en prouvant l' existence de points trans* posés de $\frac{1}{u^{5}}$, ne conduit pas immédiatement à une valeur $\eta$ de la racine cherchée. C'est ce qui est atteint seulement par le procédé suivant, d'ailleurs plus simple que l'emploi de la formule (10). Remarquons d'avance qu'il n'y 
a qu une seule approximation, en faisant usage des points transposés de $\frac{1}{u^{5}}$. Car nne approximation ultérieure réconduirait au point primaire. En second lieu, comme la valeur approchée $\left(\eta_{0}\right)$, considérée dans ce qui précède, s' en va des formules définitives, on pourra déjà d'avance supposer $\left(\eta_{0}\right)=1$ et écrire $\eta=\approx$

par où notre équation secondaire

$$
z^{5}+58 z=4 \tau
$$

soit assujettie aux conditions

$$
s=\frac{1}{u^{3}} ; \quad \tau=\frac{\frac{1}{u^{5}}-27}{4} ; \quad x=\frac{1}{\left(u^{5}\right)^{3}}\left(\frac{4^{4}}{\left(\frac{1}{u^{5}}-27\right)}=\frac{s^{5}}{\tau^{4}} .\right.
$$

Nous allons au surplus introduire quelques désignations améliorées définitives.

Soit

$$
w^{4}+4 \tau w=3 s^{5 / 3}
$$

Ia résolvante de notre equation en $z$ et mettons, afin de réduire à la forme libre,

$$
u_{0}=\frac{\left(\frac{w}{3}\right)^{4 / s}}{s^{1 / s}} ; \quad z=\zeta_{0} \frac{s^{1 / s}}{\left(\frac{w}{3}\right)^{1 / 5}},
$$

par' où s'obtient

$$
\zeta_{0}^{5}+5 u_{v} \zeta_{0}=1-27 u_{0}^{5}
$$

où bien l'équation, obtenue par la substitution

$$
\eta_{0}=\frac{\zeta_{0}}{u_{0}}
$$

suivante

$$
\eta_{0}^{5}+\frac{5 \eta_{0}}{u_{0}^{3}}=\frac{1}{u_{0}^{5}}-27
$$

tout analogue, du reste, à l'equation originelle, mais au paramètre transposé $\frac{1}{u_{0}}$.

SOLUTLON DE LA RÉSOLVANTE: $w^{1}+4 \tau w=3 s^{5 / 3}$. - Il sera commode de mettre

de manière que

$$
w=z_{1} ; \quad \tau=s_{1} ; \quad s^{3 / s}=\tau_{1}
$$

$$
z_{1}^{4}+4 s_{1} z_{1}=3 \tau_{1}
$$


à quoi appartient la résolvante du troisième degré:

$$
w_{1}^{3}+3 \tau_{1} w_{1}=2 s_{1}^{2} .
$$

Afin de réduire l'équation en $z_{1}$ du quatrième degré à la forme libre, nous aurons à employer les substitutions

$$
u_{1}=\frac{\left(\frac{w_{1}}{2}\right)^{3 / 4}}{s_{1}{ }^{1 / 2}} ; \quad z_{1}=\frac{s_{1}{ }^{1 / 2}}{\left(\frac{w_{1}}{2}\right)^{1 / 4}} \cdot \zeta_{1},
$$

d'où s'obtient

$$
\zeta_{1}^{4}-4 u_{1} \zeta_{1}=1-4 u_{1}^{\ddagger}
$$

équation qui, au moyen de la substitution

$$
\eta_{1}=\frac{\zeta_{1}}{u_{1}}
$$

se partage en deux équations du second degré, qui à leur tour possèdent les racines

$$
\eta_{1}=-1+\sqrt{\frac{1}{u_{1}^{2}}-1} ; \quad \eta_{1}=+1+i \sqrt{\frac{1}{u_{1}^{2}}+1}
$$

C'est, dans notre cas, la solution

$$
\eta_{1}=-1+\sqrt{\frac{1}{u_{1}^{2}}-1}
$$

$q u$ 'il faut employer. Le signe minus de la racine carrée réconduirait au point du départ. Les racines womplexes conduisent à des points étrangers.

SOLUTTON DE LA RÉSOLVANTE: $w_{1}^{3}+3 \tau_{1} w_{1}=2 s_{1}^{2}$. - En ayant recours aux désignations de $\tau_{i}$, et $s_{1}$, introduites ci-haut, nous trouverons

où l'on aura enfin

$$
w_{1}=\tau^{2 / 3} R
$$

$$
R=[1+\sqrt{1+x}]^{1 / s}+[1-\sqrt{1+x}]^{1 / s}
$$

RÉsumé des formules PoUR L'Équation: $\eta^{5}+\frac{5 \eta}{u^{3}}=\frac{1}{u^{5}}-27$. Selon ce que nous avons mentionné, le procédé va s'ordonner comme il suit.

Point de départ:

$$
x=\frac{1}{\left(u^{5}\right)^{3}} \cdot \frac{4^{4}}{\left(\frac{1}{u^{5}}-27\right)^{4}} ; \quad \tau=\frac{\frac{1}{u^{5}}-27}{4} .
$$


On trouvera successivement:

$$
\begin{aligned}
R & =[1+\sqrt{1+x}]^{1 / 3}+[1-\sqrt{1+x}]^{1 / 3}, \\
\frac{1}{u_{1}^{2}} & =\left(\frac{R}{2}\right)^{-s / 3}, \quad \eta_{1}=-1+\sqrt{\frac{1}{u_{1}^{2}}-1,} \\
\text { (A) } \frac{1}{u_{0}^{5}} & =81 \cdot \frac{x^{1 / s}}{\eta_{1}^{4}\left(\frac{R}{2}\right)^{2}}, \quad \text { (B) } f=\frac{\tau}{27} \cdot \eta_{1}^{3} \cdot\left(\frac{R}{2}\right)^{3 / 2},
\end{aligned}
$$

après quoi la racine cherchée est donné sous la forme

$$
\eta=f^{1 / 5} \cdot \eta_{0} \text {. }
$$

La détermination de $\eta_{0}$ sera effectuée selon l'équation

$$
\eta_{0}^{\overline{5}}+\frac{5 \eta_{0}}{u_{0}^{3}}=\frac{1}{u_{0}^{5}}-27
$$

en faisant usage du développement racinal [voir (2) ou (D) ci-bas].

Si le point $\frac{1}{w^{5}}$ considéré se trouvait dans le domaine interne, où plusieurs singularités et divergences des valeurs obtennes à l'emploi immédiat de la formule (2) ont lieu, le point transposé $\frac{1}{u_{0}^{5}}$ sera au contraire en général situé dans le domaine externe ou racinal, où le développement racinal, c.-à-d.

$$
\eta_{0}=\frac{1}{u_{0}} A-\frac{A^{2}}{p}-u_{0} \frac{p}{A^{2}}(1-s)-u_{0}^{2} \frac{1}{A}(1-s),
$$

conduit, en général, à la valeur juste de la racine $\eta_{0}$.

En général il suffit de calculer la quantité $A$ dans la formule pour $\eta_{0}$ d'après l'expression originelle (3) :

$$
A_{\delta}^{5}=1+6 \delta+22 \delta^{2}+36 \delta^{3}+22 \delta^{4}+6 \delta^{5}+\delta^{6},
$$

de laquelle ne diffère, en général, que peu l'expression secondaire (4):

$$
A_{s}^{5}=1+6 s+16 s^{2}+52 s^{3}+16 s^{4}+6 s^{5}+s^{6}
$$

où

$$
s=\gamma-2 \gamma^{2}+4 \gamma^{3}-3 \gamma^{4}
$$

La formule générale donnant $\eta_{0}$ est pourtant, selon (5):

$$
\eta_{0}=\frac{\eta_{\delta}+p_{s} \eta_{s}}{1+p_{s}}
$$

$\eta_{\delta}$ et $\eta_{s}$ désignant les valeurs obtenues suivant que la formule (b) où $\left(b_{4}\right)$ est employée. 
Pour la quantité $p_{s}$ fut proposée la formule (6), déstinée soit au point $\frac{1}{u^{5}}=27$ soit au point $\frac{1}{u^{5}}=3$. Mais, parceque le dernier n' appartient pas aux points transposés, il n'est plus nécessaire d'avoir recours à la formule générale (6), qui, de plus, ne s'étend au point second spécial de notre équation, savoir $\frac{1}{u^{5}}=-9$, que par la condition que, pour lui, $A_{s}$ soit échangée en $A_{s_{1}}$ où $s_{1}$ se déduit de la formule inverse

$$
\gamma=s_{1}+2 s_{1}^{2}+4 s_{1}^{3}+3 s_{i}^{4} .
$$

Dans ces eireonstances il parait que la valenr simple de

$$
p_{s}=\frac{1}{3} ; \quad \lg p_{s}=9.5228787
$$

soit à adopter pour les points transposés, de manière qu'il fandra bien mettre:

$$
\eta_{0}=\frac{\eta_{s}+\frac{1}{3} \gamma_{i s}}{1+\frac{1}{3}} .
$$

Si le point considéré se trouvait déjà dans le domaine externe, la transformation secondaire le conduirait au mème point, ou, en prenant le signe minus de la racine carrée, au domaine intérieure. Il va sans dire qu'alors le développement racinal (2) doit être immédiatement employé.

RHMARque. - Il faut rappeler que ce n'est qu'à l'aide d' nne formule spéciale (5) ou (8-a), et en utilisant à la foi les deux expression $A_{\delta}$ et $A_{s}$, qu' on parvient pour l'axe réel depuis $\frac{1}{u^{5}}=3$ à $\frac{1}{u^{5}}=27$ à une représentation de la racine d'accord plus marqué. C'est cela pour la partie interne de l'axe réel. Puisque les arguments sont transposés an domaine externe, e'est cependant qui ne sera pas d'importance. Les écarts ront en diminuant quand on s'approche de point $\frac{1}{u^{5}}=27$, point de racine zéro, à considérer comme point limite des domaines interne et externe. $\dot{A}$ ce point même il existe une petite différence de $A_{\delta}$ et $A_{s}$, nécessitant l'emploi de la formule de secours (5) ou (c) pour arriver à la valeur zéro de la racine. Il faut présumer que les écarts an delà du point $\frac{1}{u^{5}}=27$ soient de plus en plus presque insensibles, mais que pourtant ils puissent être de caractère systématique. Si à quelque instant l'emploi de plus de sept décimales parait désirable, on peut avoir recours 
à l'équation originelle $z^{5}+\check{\partial} s z=4 \tau$, afin d'obtenir par une approximation ultérieure faite sur cette formule un nombre de chiffres plus grand. Parceque en tout cas il faudra contròler les calculs, en ayant recours à l'équation initiale, e'est qui se fera sans difficulté. Mais, nous n'insisterons pas ici de plus sur cette question.

10. Exemples. - Le cas de racines égales fut déjà exposé dans ce qui précède. Nous allons considérer le cas des isolines, où la singularité la plus essentielle dans le domaine intérieure a lieu. Ces points sont

$$
\frac{1}{u^{5}}=+15.2329+i 10.4949
$$

On obtient dans ce cas

$$
\begin{gathered}
x=+0.2814-i 26.216, \quad x^{1 / 3}=\left[8.02646_{n}\right]+i[0.47286] \\
R=-0.000206-i 0.22434
\end{gathered}
$$

et il suffira de mentionner encore les résultats suivants

$$
\begin{gathered}
\frac{1}{u_{0}^{5}}=56.830+i 25.394, \quad f=\frac{\tau}{27} \gamma_{i 1}^{3}\left(\frac{R}{2}\right)^{3 / 2}=\left[8.74099_{n}\right]+i[9.60069], \\
f^{1 / 5}=\left[8.35953_{n}\right]+i[9.92080], \quad \eta_{0}=[9.77197]+i[9.45598],
\end{gathered}
$$

d'où s'ensuit

$$
\eta=-0.25164+i 0.48639, \quad \text { valeur vraie }:-0.25161+i 0.48639,
$$

l'accord étant ainsi établi.

On obtient, en utilisant la deuxième racine

le mème point transposé

$$
R_{2}=2.12017-i 1.99063
$$

$$
\frac{1}{u_{0}^{5}}=+56.830+i 25.394
$$

mais à l'emploi de la troisième racine

$$
R_{3}=-2.11821+i 2.21497,
$$

aucun résultat utile n'est trouvé.

Si nous écrivons d'une manière schématique

$$
R=(+)^{1 / 3}+(-)^{1 / 3} \quad \text { et } \quad(+)=p e^{i \vartheta} \quad \text { et } \quad(-)=p e^{i \hat{\vartheta}},
$$

on aura à appliquer à $\frac{9}{3}$ dans l'expression de

$$
R=(+)^{1 / 3}+(-)^{1 / 3},
$$

$240^{\circ}$ et $0^{\circ}$ pour le premier terme et pour le second respectivement. 
On aura les corréspondances suivantes:

$$
\begin{gathered}
\frac{\vartheta}{3}:+240^{\circ} ;+0^{\circ} \\
\eta_{1}: \quad-\quad+ \\
\frac{1}{u_{0}^{5}}=56.830+i 25.39 \\
\text { point transposé } \\
\vartheta^{\vartheta}:+0^{\circ} ;+240^{\circ} \\
3 \quad- \\
\eta_{1}:-\quad-15.23+i 10.49 \\
\frac{1}{u_{0}^{5}}=+19 \text { printive }
\end{gathered}
$$

$$
\begin{gathered}
\frac{9}{3}:+240^{\circ} ;+0^{\circ} \\
\eta_{1}:- \\
\frac{1}{u_{0}^{5}}=14.20+i 23.03 \\
\text { point contraposé } \\
\frac{9}{3}:+0^{\circ} ;+240^{\circ} \\
\eta_{1}: \quad-\quad+ \\
\frac{1}{u_{0}^{5}}=56.83+i 25.39
\end{gathered}
$$

point transposé.

A la détermination de la racine dans les isolines appartient encore la quantité $\eta_{0}$, devant être calcalée par le racinal (2) de l'equation

$$
\eta_{0}^{5}+\frac{\check{\partial} \eta_{0}}{u_{0}^{3}}=\frac{1}{u_{0}^{5}}-27
$$

dans le point transposé

$$
\frac{1}{u_{0}^{5}}=+56.830+i 25.395
$$

Il n'y a lieu de réproduire des calculs que quelques traits principaux. On a obtenu

$$
\alpha=-0.045587+i 0.021198
$$

et puis les valeurs corréspondentes de $\beta, \gamma, \delta, s, p$, pour enfin parvenir à la valeur $A_{\delta}^{5}$

$$
A_{\delta}^{5}=+0.912734+i 0.038368 \text {. }
$$

Ensuite s'obtient, au moyen de la formule

$$
\eta_{0}=\frac{1}{u_{0}} A-\frac{A^{2}}{p}-u_{0} \frac{p}{A^{2}}(1-s)-u_{0}^{2} \frac{1}{A}(1-s),
$$

la valeur cherchée

$$
\eta_{0}=\eta_{\delta}=+0.5915 \overline{5}+i 0.28575
$$

c'est la valeur inscrite ci-haut dans la première partie des calculs.

ExEMPLe : $\frac{1}{u^{5}}=7$. On aura dans ce cas

$$
x=\frac{7^{3}}{5^{4}} \quad \text { et } \quad \tau=-5
$$


et l'on obtient de plus

$$
\frac{1}{u_{1}^{2}}=+5
$$

et pour $\eta_{1}$ les quatre valeurs

$$
+1, \quad-3, \quad+1+i \sqrt{6}, \quad+1-i \sqrt{6},
$$

desquelles la première est à employer. Le point transposé devient

$$
\frac{1}{u_{0}^{5}}=7.81
$$

et dans ce point on trouve

$$
\eta_{0}=0.339318
$$

de quoi enfin

$$
\eta=-1.129914, \quad \text { valeur vraie: }-1.129915 \text {. }
$$

EXEMPle: $\frac{1}{u^{5}}=8$. - Le calcul fournit le point transposé

$$
\frac{1}{u_{0}^{5}}=+350.6905
$$

puis, dans ce point,

$$
\eta_{0}=1.8088863
$$

et enfin, pour la racine $\eta$, la valeur

$$
\eta=-1.025973, \quad \text { valeur vraie: } 1.025972 \text {. }
$$

Aussi confirmées sont les formules pour les points

$$
\frac{1}{u^{5}}=3 ; \quad \frac{1}{u^{5}}=-9 ; \quad \frac{1}{u^{5}}=16 ; \quad \frac{1}{u^{5}}=21 ; \quad \frac{1}{u^{5}}=36,
$$

et d'autres qui furent considérés de plus on de moins.

11. Sur le choix des signes et des quadrants. - Dans les formules figurent des racines telles que

$$
\text { 10) } \left.x^{1 / s} ; \quad 20\right) f^{1 / 5} \text {. }
$$

Dans le cas de quantités réelles il n'y a pas de difficulté par rapport à cela. Dans les cas complexes, comme il s'agit d'une racine principale, on aura, pour $x^{1 / 3}$ et $f^{1 / 5}$, a appliquer des valeurs convenables de $\frac{2 k \pi}{3}$ et $\frac{2 k \pi}{5}$. Nous pouvons choisir les $k$ de manière que les racines soient homologues aux valeurs $\%$ et $f$ mêmes. Il peut arriver qu' on aura les mèmes quadrants, mais aussi que les quadrants soient opposés. Soient $a+b i$ les expressions 
des quantités mèmes et $\alpha+\beta i$ les expressions de leurs racines. Ce qu'on doit exiger de $\alpha, \beta$, c'est qu'elles s'accomodent de façon homologue aux quantités $a, b$, les quadrants devant ètre les mêmes ou bien les opposés, ce qui sera en général autrement pour les racines troisièmes et pour les cinquièmes.

Quant au choix des quadrants dans l'expression binome

$$
3^{(1)} \quad R=[1+\sqrt{1+x}]^{1 / s}+[1-\sqrt{1+x}]^{1 / s},
$$

nous nous avons déjà prononcé dans ce qui précède. Il faudra les choisir de manière que des résultats étrangers soient évités. Nous n'entrerons pas ici sur cette question en général. Dans le cas des isolines, le choix juste se trouve indiqué par ce qu'on peut y arriver de deux manières différentes. 